

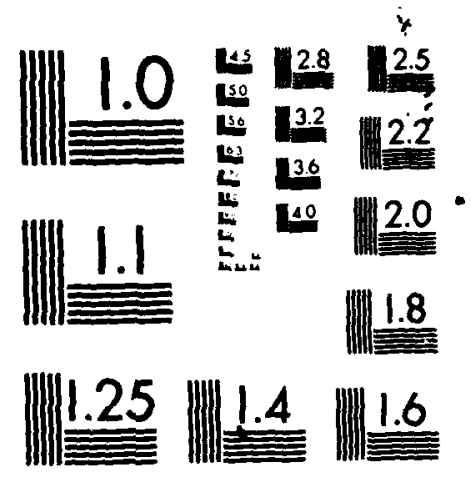

MICROCOPY RESOLUTION TEST CHART

NATIONAL BUREAU OF SIANDARDS.1963.A 


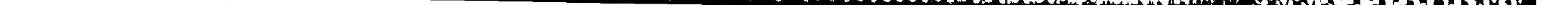


secuaitr classification of this page

Uly cont.

Theat release for a given incident turbulence intensity increases the amolitude of the flame fluctuations without modifying their spectral characteristics.

A study not yet completed and being pursued under the contihudition of this grant is the investigation of the structure of the reaction region just downstream of a fiameholder and its evolution into a thin flame sheet. 


\title{
AFOSR:TR. $86 \cdot 0881$
}

\author{
FINAL TECHNICAL REPORT

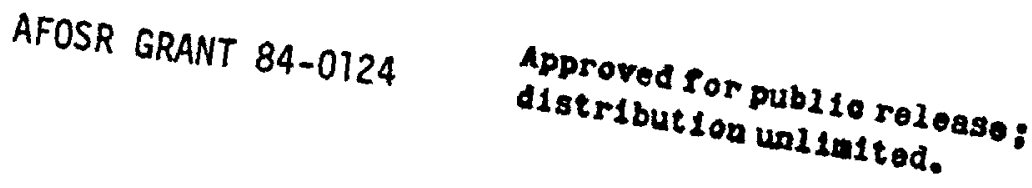 \\ APPLICATION OF RAYLEIGH SCATTERING TO \\ TURBULENT FLOW WITH HEAT TRANSFER ANO COMBUSTION \\ Principal In \\ University of Californ: L. Talbot \\ of California, Berkeley
}

June 9, 1986

AIR FORCE OFFICE OF SCIENTIFIC RESEARCH (AFSC)

MOTICE OR TRANSMITTAL TO DTIC

This tochnical report has been rovlewnd and is

coproved for public re ? e so IAW AFR 190-12.

i'strifution ir: unlimited.

"THEF $I$ Y Y T

3. Ter $\cdots 1$ Information Division

The Ter

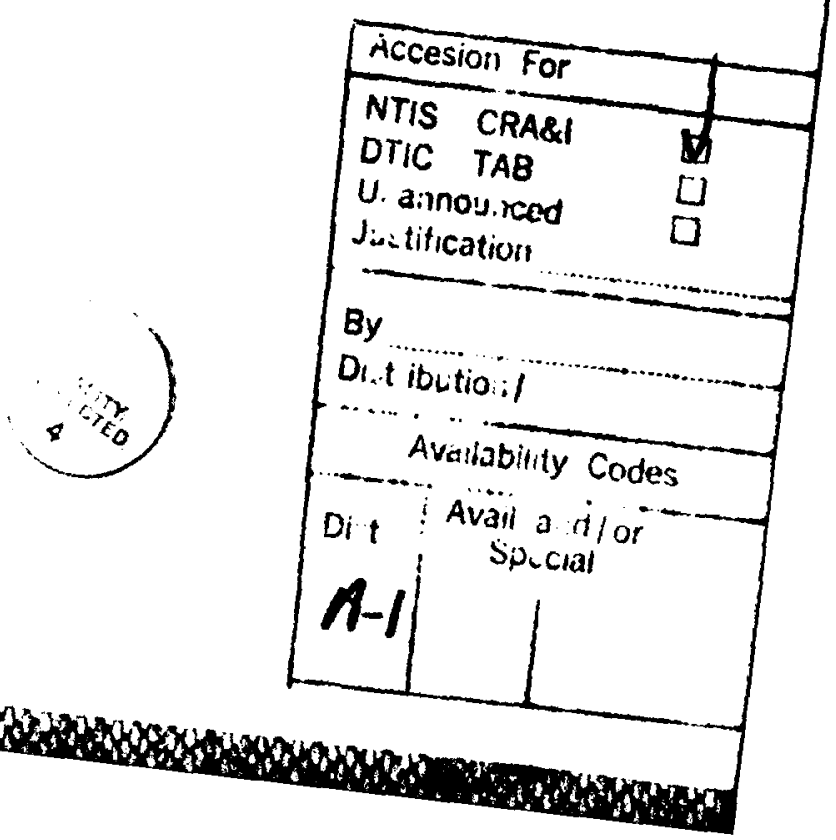


RESEARCH OBJECTIVES

The objectives of this research program has been to investigate experimentally the fluid mechanical properties of premixed turbulent flames, through detailed measurements and flow visualization techniques. The combustion configuration investigated is a $v$-shaped, unconfined, rodstabilized flame propagating into a turbulent, essentially uniform flow. Laser-based diagnostics for the measurement of instantaneous density through Rayleigh scattering, and velocity through Doppler velocimetry are the principal quantitative experimental techniques, while laser-tomographic high speed cinematographic flow visualization provides qualitative information on the behavior of the flame. The research has focused on two central goals: to determine (a) the detailed structure underlying that which in the large constitutes a turbulent flame brush; and (b) to what extent this structure can be described theoretically by models such as the Bray-MossLibby (BML) model.

\section{RESEARCH RESULTS}

During the period of this grant, several experimental programs were pursued.

1. Two-point Rayleigh scattering measurements within the turbulent flame brush of ethylene-air and methane-a ir $v$-flames were extended to provide more extensive information on such features of the reaction zone as the spectral distribution of the density fluctuations, their autocorrelation functions and integral scales, and mean density profiles. Some of the findings include:

a) The mean density profiles through the reaction zone at different distances downstream of the flame holder are self-similar when scaled by the profile maximum slope thickness which is a function of the equivalence 
ratio and the upstream turbulence intensity, for a given reactant. This maximum slope thickness is found to grow linearly with distance from the flame holder. The probability function for the location of the wrinkled laminar flame sheet within the turbulent flame brush was found to be accurately represented by a Gaussian function.

b) The probability density of 'intermediate states' was measured at various locations downstream of the flame holder. Contrary to the assumptions generally employed in the BML model, the contribution to the overall propability density function ( $p d f$ ) contains a significant contribution from the intermediate states, rather than being composed of two delta functions associated with the reactants and products. An extension of the BML model to take account of the intermediate states, which are due to the finite thickness of the wrinkled laminar flame sheet, was developed and shown to be in good agreement with the measured density fluctuation intensities and covariances. This extension of the BML model of the pdf also provides a means for introducing finite-rate chemistry into the production term of the model, although this has yet to be tested.

The work described above has been reported in References 1 and 2. 2) Studies of optical techniques for the visualization of turbulent flame structure have been pursued using two approaches. In this first approach, which was initiated under our previous. AFOSR Grant F49620-80-C-0065 and which we have called 'laser tomegraphy', the unburned gas is seeded with a silicone oil aerosol which is illuminated by the scattering of a sheet of laser light. The aerosol evaporates in passing through the flame, thus rendering the burned gas transparent to the light and producing a light-dark boundary which coincides with the instantaneous position and shape of the flame front. High speed cinematography of the illuminated flow region then yields a time-resolved record of the motion of the flame front. 
The method was used to study the interaction of a Karmán vortex street with rod-stabilized $V$-shaped laminar flame. The wavelength and frequency of the flame motion were found to correspond closely to that of the vortex street, allowing a simple phenomenological explanation of the cyclic behavior of the flame. Particularly interesting was the observation of the formation and evolution of cusps in the flame front, and how they subsequently are erased by the propagation of opposing flame surfaces toward one another.

The success of these qualitative tomographic studies encouraged us to move toward a more quantitative flow visualization technique, the use of a rapidly scanned linear diode array on which was imaged the Rayleighscattered light produced by a laser beam projected through the flame. The technique, to which we have given the name LARS (Linear Array for Rayleigh Scattering), was implemented under DoD-University Research Instrumentation Grant AFOSR 83-0229, and is described in detail in the Final Technical Report for this grant (Ref. 4). Much of the effort during the period of the present grant was directed toward the 'fine-tuning' of the LARS system, and then to its application in the study of the dynamics of turbulent flame fronts.

The first quantitative application of the LARS system has been to the study of the same types of flames investigated earlier (Refs. 1 and 2) by two-point Rayleigh techniques. Methane-air premixed turbulent V-shaped flames at an approach velocity of $2 \mathrm{~m} / \mathrm{s}$ and a range of inlet turbulence levels and equivalence ratios from $5 \%-8 \%$ and $0.6-0.8$ respectively were studied. The statistics of the flame front position were obtained by extracting the flame boundary from the images and generating a pdf of its distance from the mean. The standard deviation of these distributions varied from $0.31 \mathrm{~mm}$ to $1.38 \mathrm{~mm}$ and show that increases in inlet turbulence 
and equivalence ratio thicken the turbulent flame zone by amplifying the flame motions. These results lend support to various modeling assumptions. Comparison of the spectra of the flame front oscillations and the cold flow velocity indicate that the incident turbulence controls flame motion at frequencies between 100 and $.1000 \mathrm{~Hz}$. Changes in heat release for a given inlet turbulence increase the amplitude of the flame fluctuations without modifying the spectral characteristics.

A paper describing this work (Ref. 5) has been accepted for presentation at the forthcoming Twenty-First (International) Symposium on Combustion, Munich, 1986.

3. Experiments now in progress are being carried out to investigate the structure of the reaction zone close to the flame holder, the object being to determine how the flame stabilization and recirculation zone evolves into a thin flame sheet. It appears likely that this region cannot be adequately described by the Bray-Moss-Libby model, even in the 1 imit very fast chemistry. Since in this transition region density changes can be the result of convection/conduction heat transfer as well as combustion heat release, the location of the reaction zone cannot be determined uniquely by Rayleigh scattering measurements and, as will be described, must be supplemented by other diagnostics.

The combustor configuration for this study is the same as for previous $v$-flame studies with the exception that flame holder is either a $6 \mathrm{~mm}$ diameter rod or a bar with $6 \mathrm{~mm} \times 3 \mathrm{~mm}$ cross-section. The bar provides a well defined flow separation point and good optical access to the immediate downstream wake region. The rod was chosen for comparison with other $v$-flame data. Two conditions have been studied: a very lean flame, close to being blown off the flameholder at $6 \mathrm{~m} / \mathrm{s}$, equivalence ratio $\phi=0.54$; and a slightly richer, well stabilized flame at $6 \mathrm{~m} / \mathrm{s}, \phi=.63$. 
Point measurements of density using laser Rayleigh scattering have been made in a region beginning at $0.5 \mathrm{~mm}$ downstream of the flameholder and extending to $80 \mathrm{~mm}$ downstream. Flame brush extent and position have been calculated from time averaged profiles using the steepest gradient method. The well stabilized flame has a nearly constant rate of brush growth, agreeing with other $v$-flame data, while the very lean case shows a much more rapid growth for the first $25 \mathrm{~mm}$ and then slows down for the rest of the observed region, possibly indicating increased interaction with the recirculation zone.

To examine the evolution of the structure of the brush with distance downstream the probability density function (pdf) of gas density at the center of the flame has been calculated at several locations. Results show that close to the flameholder the richer case has the bimodal pdf characteristics of wrinkled laminar flames, while the very lean case, although it has a thicker flame brush, has a broad monomodal pdf. Further downstream the very lean case also develops a bimodal pdf at the center of the flame.

Detailed two-component LDV velocity field measurements have been made recently for both flameholders at both equivalence ratios, plus isothermal conditions. In addition, conditioned measurements have been made by using silicone oil droplets for seed, which disappear in the flame, conditioning the signal by reporting only unburned gas velocities. Spectral data of transverse velocity fluctuations have also been obtained. Time averages of mean, rms and covariance of axial and transverse (perpendicular to the flameholder) velocities have been calculated.

As noted, the pdf of the beginning region of the reaction zone in the lean combustion case is monomodal, and consequently a unique flame front location cannot be specified by Rayleigh scattering measurements alone. Under the continuation of this Grant, we will complete this investigation 
6

by utilizing ionization probe studies to provide additional information on the evolution of the reaction zone into a thin flame sheet. Another possibility which will be explored is the use of laser-induced fluorescence measurements of $\mathrm{CH}$ concentration, as described in the continuation proposal for this grant, to locate more precisely the position of the flame front. Reference 6 represents a progress report on this work. 


\section{REFERENCES}

1. M. Namazian, L. Talbot and F. Robben, "Density Fluctuations in Premixed Turbulent Flames", Twentieth Symposium (International) on Combustion/The Combustion Institute 1984, pp. 411-419.

2. M. Namazian, I.G. Shepherd and L. Talbot, "Characterization of the Density Fluctuations in Turbulent V-Shaped Premixed Flames", presented at the Western States Section/The Combustion Institute, 22-23 October 1984, Stanford University. Accepted for publication in Combustion and Flame.

3. J.R. Hertzberg, M. Namazian and L. Talbot, "A Laser Tomographic Study of a Laminar Flame in a Kármán Vortex Street", Combustion Science and Technology, Vol. 38, 1984, pp. 205-216.

4. L. Talbot, "A Linear Array for Rayleigh Scattering", Final Technical Report, AFOSR Grant 83-0229, June 11, 1985.

5. I.G. Shepherd, G.L. Hubbard and L. Talbot, "The Dynamic Structure of Turbulent V-Shaped Premixed Flames", accepted for presentation at the Twenty-First Symposium (International) on Combustion/The Combustion Institute, Munich, August 3-8, 1986.

6. J. R. Hertzberg and L. Talbot, "Premixed Flame Stabilization on a Bluff Body": accepted for presentation (poster session) at the Twenty-First Combustion Institute, Munich, August 3-8, 1986. 


$$
\begin{aligned}
& \text { END } \\
& 11-86 \\
& \text { DTIC }
\end{aligned}
$$

